\title{
A short proof of chaos in an atmospheric system
}

\author{
Petre Birtea $^{\mathrm{a}, *}$, Mircea Puta ${ }^{\mathrm{b}}$, Tudor S. Ratiu ${ }^{\mathrm{a}}$, Răzvan Tudoran ${ }^{\mathrm{a}}$ \\ a Institut Bernoulli, École Polytechnique Fédérale de Lausanne, CH-1015 Lausanne, Switzerland \\ ${ }^{\mathrm{b}}$ Departamentul de Matematică, Universitatea de Vest, 1900 Timişoara, Romania \\ Received 12 March 2002; received in revised form 12 March 2002; accepted 18 June 2002 \\ Communicated by A.P. Fordy
}

\begin{abstract}
We will prove the presence of chaotic motion in the Lorenz five-component atmospheric system model using the Melnikov function method developed by Holmes and Marsden for Hamiltonian systems on Lie Groups. C 2002 Elsevier Science B.V. All rights reserved.
\end{abstract}

PACS: 02.20.Sv; 02.30.Hg; 02.40.-k; 92.60.-e

Keywords: Dynamical system; Poisson bracket; Melnikov function; Chaos

\section{Introduction}

The first model equations for the atmosphere are the so-called primitive equations (PE). This model allows wave-like motions on different time scales. The slow motions which have a period of order of days are called Rossby waves and the fast motions which have a period of hours are called gravity waves. The question of how to balance these two time scales lead Lorenz [5] to introduce a simplified version of the (PE) model, the so-called five-component model. This is a system of five differential equations which couples the Rossby waves and gravity waves. This system turns out to have a Poisson formulation on $\mathbb{R}^{5}$ first discovered by Bokhove [1]. We shall find a Poisson diffeomorphism between the Poisson structure of Bokhove and the product structure on $\mathfrak{s e}^{*}(2) \times \mathbb{R}^{2}$, where the

\footnotetext{
* Corresponding author.

E-mail address: petre.birtea@epfl.ch (P. Birtea).
}

first factor is endowed with the Lie-Poisson structure and the second with the standard symplectic structure. Using this diffeomorphism, the five-component Lorenz model takes on a form appropriate for the application of the Melnikov function method on Lie groups developed by Holmes and Marsden [4] in order to study the presence of chaotic motion. The presence of chaos for the five-component Lorenz model was first proved by Camassa in [3], see also [2].

\section{The geometry of Lorenz simplified model of Rossby gravity-wave interaction}

The model introduced by Lorenz in [5] is described by the following set of differential equations:

$$
\begin{aligned}
& \dot{x}_{1}=-x_{2} x_{3}+\varepsilon x_{2} x_{5}, \quad \dot{x}_{2}=x_{1} x_{3}-\varepsilon x_{1} x_{5}, \\
& \dot{x}_{3}=-x_{1} x_{2}, \quad \dot{x}_{4}=-x_{5}, \\
& \dot{x}_{5}=-x_{4}+\varepsilon x_{1} x_{2},
\end{aligned}
$$


where the variables $x_{4}, x_{5}$ represent the fast gravity wave oscillations and $x_{1}, x_{2}, x_{3}$ are the slow Rossby wave oscillations, with the parameter $\varepsilon$ (related to the Rossby number) coupling the two sets of variables.

In [1], Bokhove wrote the system (2.1) in the following Hamiltonian form:

$\dot{x}=\{x, H\}_{1}$,

where the Hamiltonian function is given by

$$
\begin{aligned}
& H\left(x_{1}, x_{2}, x_{3}, x_{4}, x_{5}\right) \\
& \quad=\frac{1}{2}\left(x_{1}^{2}+2 x_{2}^{2}+x_{3}^{2}+x_{4}^{2}+x_{5}^{2}\right),
\end{aligned}
$$

and the Poisson bracket $\{\cdot, \cdot\}_{1}$ is defined by

$$
\begin{aligned}
\{f, g\}_{1}= & x_{1}\left[\left(\frac{\partial f}{\partial x_{2}} \frac{\partial g}{\partial x_{3}}-\frac{\partial f}{\partial x_{3}} \frac{\partial g}{\partial x_{2}}\right)\right. \\
& \left.+\varepsilon\left(\frac{\partial f}{\partial x_{5}} \frac{\partial g}{\partial x_{2}}-\frac{\partial f}{\partial x_{2}} \frac{\partial g}{\partial x_{5}}\right)\right] \\
+ & x_{2}\left[\left(\frac{\partial f}{\partial x_{3}} \frac{\partial g}{\partial x_{1}}-\frac{\partial f}{\partial x_{1}} \frac{\partial g}{\partial x_{3}}\right)\right. \\
& \left.+\varepsilon\left(\frac{\partial f}{\partial x_{1}} \frac{\partial g}{\partial x_{5}}-\frac{\partial f}{\partial x_{5}} \frac{\partial g}{\partial x_{1}}\right)\right] \\
+ & \frac{\partial f}{\partial x_{5}} \frac{\partial g}{\partial x_{4}}-\frac{\partial f}{\partial x_{4}} \frac{\partial g}{\partial x_{5}} .
\end{aligned}
$$

On the space $\mathfrak{s e}^{*}(2) \times \mathbb{R}^{5}$ consider the Poisson bracket given by the product bracket of the Lie-Poisson bracket on $\mathfrak{s e}^{*}(2)$ and the Poisson bracket on $\mathbb{R}^{2}$ induced by the standard symplectic form on $\mathbb{R}^{2}$. Denoting by $\left(\mu_{1}, \mu_{2}, \mu_{3}\right)$ the variables on $\mathfrak{s e}^{*}(2)$ and by $\left(u_{1}, u_{2}\right)$ the variables on $\mathbb{R}^{2}$, the product Poisson bracket is given by

$$
\begin{aligned}
\{f, g\}_{2}= & x_{1}\left(\frac{\partial f}{\partial \mu_{2}} \frac{\partial g}{\partial \mu_{3}}-\frac{\partial f}{\partial \mu_{3}} \frac{\partial g}{\partial \mu_{2}}\right) \\
& +\mu_{2}\left(\frac{\partial f}{\partial \mu_{3}} \frac{\partial g}{\partial \mu_{1}}-\frac{\partial f}{\partial \mu_{1}} \frac{\partial g}{\partial \mu_{3}}\right) \\
& +\frac{\partial f}{\partial u_{2}} \frac{\partial g}{\partial u_{1}}-\frac{\partial f}{\partial u_{1}} \frac{\partial g}{\partial u_{2}} .
\end{aligned}
$$

The Casimir function for this Poisson bracket is given by

$C\left(\mu_{1}, \mu_{2}, \mu_{3}\right)=\mu_{1}^{2}+\mu_{2}^{2}$.

It is easy to verify that the linear transformation $\Phi$ : $\mathbb{R}_{5} \rightarrow \mathfrak{s e}^{*}(2) \times \mathbb{R}^{2}$ given by

$\Phi\left(x_{1}, x_{2}, x_{3}, x_{4}, x_{5}\right)=\left(x_{1}, x_{2}, x_{3}, x_{4}, \varepsilon x_{3}+x_{5}\right)$ is a Poisson diffeomorphism between $\left(\mathbb{R}^{5},\{\cdot, \cdot\}_{1}\right)$ and $\left(\mathfrak{s e}^{*}(2) \times \mathbb{R}^{2},\{\cdot, \cdot\}_{2}\right)$.

In the new variables the system (2.1) becomes

$$
\begin{aligned}
& \dot{\mu}_{1}=-\mu_{2} \mu_{3}+\varepsilon \mu_{2} u_{2}-\varepsilon^{2} \mu_{2} \mu_{3}, \\
& \dot{\mu}_{2}=\mu_{1} \mu_{3}-\varepsilon \mu_{1} u_{2}+\varepsilon^{2} \mu_{1} \mu_{3}, \quad \dot{\mu}_{3}=-\mu_{1} \mu_{2}, \\
& \dot{u}_{1}=-u_{2}+\varepsilon \mu_{3}, \quad \dot{u}_{2}=-u_{1},
\end{aligned}
$$

which is an Hamiltonian system with respect to the Poisson bracket $\{\cdot, \cdot\}_{2}$ and the Hamiltonian function is given by

$$
\begin{aligned}
& H^{\varepsilon}\left(\mu_{1}, \mu_{2}, \mu_{3}, u_{1}, u_{2}\right) \\
& \quad=\frac{1}{2}\left(\mu_{1}^{2}+2 \mu_{2}^{2}+\mu_{3}^{2}+u_{1}^{2}+u_{2}^{2}-2 \varepsilon \mu_{3} u_{2}+\varepsilon_{2} \mu_{3}^{2}\right) .
\end{aligned}
$$

\section{Chaos by the Melnikov method}

We will prove the occurrence of chaotic motion in the system (2.2) by showing the existence of transverse heteroclinic orbits. In [6] Melnikov (see also [7]) gave an effective method to prove the existence of transverse heteroclinic (homoclinic) orbits in the Poincaré map for a perturbed one-degree of freedom Hamiltonian system by measuring the "distance" between the stable and unstable manifolds associated with the saddle points. This method was generalized by Holmes and Marsden to the case of perturbed twodegree of freedom Hamiltonian systems when the phase space is a product of the dual of a Lie algebra and a set of action-angle variables. We briefly recall below this result; see Holmes and Marsden [4] for proofs.

The setting is the following. The phase space is the product of the dual of a Lie algebra $\mathfrak{g}$ and $\mathbb{R}^{2}$. The Hamiltonian has the form

$$
\begin{aligned}
H^{\varepsilon}(\mu, \theta, I) & =F(\mu)+G(I)+\varepsilon H^{1}(\mu, \theta, I)+O\left(\varepsilon^{2}\right) \\
& =H^{0}(\mu, I)+\varepsilon H^{1}(\mu, \theta, I)+O\left(\varepsilon^{2}\right)
\end{aligned}
$$

where $\mu=\left(\mu_{1}, \ldots, \mu_{m}\right) \in \mathfrak{g}^{*}$ and $(\theta, I)$ are coordinates on $\mathbb{R}^{2}$, with $\theta$ a $2 \pi$-periodic variable. It is also assumed that the Lie-Poisson system whose Hamiltonian is $F$ has a heteroclinic (or homoclinic) orbit $\tilde{\mu}(t) \in \mathfrak{g}^{*}$. The oscillator frequency

$\Omega(I):=\frac{\partial G}{\partial I}$ 
is assumed to be positive. The result is the following:

Theorem 3.1 (Holmes-Marsden). Suppose $\tilde{\mu}(t)$ is a heteroclinic (or homoclinic) orbit for the Lie-Poisson system whose Hamiltonian is $F$, which lies in a twodimensional coadjoint orbit in $\mathfrak{g}^{*}$. Let $\tilde{h}=F(\tilde{\mu})$ be the energy of the heteroclinic orbit and let $h>\tilde{h}$ and $l^{0}=$ $G^{-1}(h-\tilde{h})$ be constants. Let $\left\{F, H^{1}\right\}\left(t, \theta^{0}\right)$ denote the Lie-Poisson bracket of $F(\mu)$ and $H^{1}\left(\mu, \Omega\left(l^{0}\right) t+\right.$ $\left.\theta^{0}, l^{0}\right)$ evaluated at $\tilde{\mu}(t)$. Let

$M\left(\theta^{0}\right)=\frac{1}{\Omega\left(l^{0}\right)} \int_{-\infty}^{\infty}\left\{F, H^{1}\right\}\left(t, \theta^{0}\right) d t$

and assume $M\left(\theta^{0}\right)$ has simple zeros. Then for $\varepsilon>0$ sufficiently small, the Hamiltonian system (3.1) contains transverse heteroclinic orbits and hence Smale horseshoes on the energy surface $H^{\varepsilon}=h$.

Now we will prove that the system (2.2) verifies the conditions of the above theorem. The unperturbed system of (2.2) on $\mathfrak{s e}^{*}(2)$ has unstable critical points $(0, \pm M, 0)$ lying on the 2-dimensional coadjoint orbit given by the cylinder

$\left\{\left(\mu_{1}, \mu_{2}, \mu_{3}\right) \in \mathfrak{s e}^{*}(2): \mu_{1}^{2}+\mu_{2}^{2}=M^{2}\right\}$.

On this coadjoint orbit we have the heteroclinic orbits given by

$\left\{\begin{array}{l}\mu_{1}(t)= \pm M \operatorname{sech}(M t), \\ \mu_{2}(t)= \pm M \tanh (M t), \\ \mu_{3}(t)= \pm M \operatorname{sech}(M t),\end{array}\right.$

that link the unstable critical points $(0, M, 0)$ and $(0,-M, 0)$.

On $\mathbb{R}^{2}$ the unperturbed system of (2.2) is completely integrable and in action-angle coordinates $(I, \theta), u_{1}=\sqrt{2 I} \cos \theta, u_{2}=\sqrt{2 I} \sin \theta$, takes the form $\dot{I}=0, \quad \dot{\theta}=1$.

Now writing the Hamiltonian (2.3) in the form

$$
\begin{aligned}
H^{\varepsilon}\left(\mu_{1}, \mu_{2}, \mu_{3}, I, \theta\right)= & \frac{1}{2}\left(\mu_{1}^{2}+2 \mu_{2}^{2}+\mu_{3}^{2}\right)+I \\
& -\varepsilon \mu_{3} \sqrt{2 I} \sin \theta+O\left(\varepsilon^{2}\right),
\end{aligned}
$$

we are in the setting of the Holmes-Marsden theorem and we can write the heteroclinic orbits for an energy level
$H^{0}=h=M^{2}+k$,

where $M^{2}=\mu_{1}^{2}+\mu_{2}^{2}$ and $k$ is a constant, as

$$
\left\{\begin{array}{l}
\mu_{1}(t)= \pm M \operatorname{sech}(M t) \\
\mu_{2}(t)= \pm M \tanh (M t) \\
\mu_{3}(t)= \pm M \operatorname{sech}(M t) \\
I=k, \quad \theta=t+\theta^{0}
\end{array}\right.
$$

The Melnikov function is:

$$
\begin{aligned}
& M\left(\theta^{0}\right)=-\int_{\infty}^{\infty} \sqrt{2 k} M^{2} \operatorname{sech}(M t) \tanh (M t) \\
& \times \sin \left(t+\theta^{0}\right) d t \\
&=-\sqrt{2 k} M^{2}\left(\int_{-\infty}^{\infty} \sinh (M t) \cosh ^{-2}(M t)\right. \\
&\times \sin (t) d t) \cos \theta^{0} \\
&=-\pi \sqrt{2 k} \operatorname{sech}\left(\frac{\pi}{2 M}\right) \cos \theta^{0},
\end{aligned}
$$

which has simple zeros as a function of $\theta^{0}$ and therefore the Hamiltonian system (2.2) for $\varepsilon>0$ sufficiently small, has transverse heteroclinic orbits and hence Smale horseshoes in a suitably chosen cross section of the constant energy surface with $k>0$.

\section{Acknowledgements}

This research was partially supported by the European Commission and the Swiss Federal Government through funding for the Research Training Network Mechanics and Symmetry in Europe (MASIE) as well as the Swiss National Science Foundation.

\section{References}

[1] O. Bokhove, On Hamiltonian balanced models, in: 9th Conf. on Atmospheric and Oceanic Waves and Stability, San Antonio, TX, Amer. Meteor. Society, 1993, pp. 367-368.

[2] O. Bokhove, T.G. Shepherd, J. Atmos. Sci. 53 (1996) 276.

[3] R. Camassa, Physica D 84 (1995) 357.

[4] P.J. Holmes, J.E. Marsden, Indiana Univ. Math. J. 32 (1983) 273.

[5] E.N. Lorenz, J. Atmos. Sci. 43 (1986) 1547.

[6] V.K. Melnikov, Trans. Moscow Math. 12 (1963) 1.

[7] S. Wiggins, Global Bifurcations and Chaos, Springer-Verlag, New York, NY, 1988. 